\title{
ANÁLISE DA INCIDÊNCIA DA MORTALIDADE POR TUBERCULOSE NO ESTADO DE PERNAMBUCO NO PERÍODO DE 2015 A 2017
}

\author{
ANALYSIS OF THE INCIDENCE OF TUBERCULOSIS MORTALITY IN \\ THE STATE OF PERNAMBUCO IN THE PERIOD FROM 2015 TO 2017
}

\author{
Fabiana de Araújo Lopes ${ }^{1}$ \\ Ozenilda Roberta de Amorim ${ }^{2}$ \\ Lannara de Melo Ferreira ${ }^{3}$ \\ Ana Cecília Amorim de Souza ${ }^{4}$
}

RESUMO: OBJETIVO: Analisar a incidência de óbitos por tuberculose no estado de Pernambuco entre 2015 a 2017. MÉTODO: Por meio do banco de dados do Sistema de Informação de Agravos de Notificação (SINAN), foram avaliadas as fichas de notificação para tuberculose no estado de Pernambuco de 2015 a 2017, fornecido pelo DATASUS/Ministério da Saúde. A tabulação dos dados no Departamento de Informática do Sistema Único de Saúde (DATASUS) foi realizada através do SINAN, que é alimentado pela notificação e investigação de casos de doenças e agravos que constam da lista nacional de doenças de notificação compulsória. Foi construído um Banco de Dados no Programa Estatístico SPSS versão $22 \mathrm{com}$ as variáveis incluídas no estudo. Para análise desses dados, foram utilizadas cálculos de porcentagem para observar a dispersão entre as variáveis coletadas, analisadas através de percentual simples. As informações foram analisadas por meio do programa SPSS versão 22 para a formatação dos dados. RESULTADOS: Observou-se uma totalidade de 16.933 casos notificados de tuberculose no estado de Pernambuco no período de 2015 a 2017. O município com maior índice da doença foi Recife, caracterizando $45,75 \%$. Os resultados mostraram que o sexo mais acometido é o masculino (70,24\%), a faixa etária mais acometida foi entre 20 e 39 anos $(46,44 \%)$, a raça parda predominou em números de casos $(43,94 \%)$ e evolução para o óbito $(5,82 \%), 86,32 \%$ dos casos foram classificadas como sendo a forma pulmonar, e apenas $2,06 \%$ eram portadores das duas formas, pulmonar e extrapulmonar. CONCLUSÃO: Com os altos números de casos confirmados de

\footnotetext{
1 Acadêmica do curso de Enfermagem da Faculdades Integradas da Vitória de Santo Antão FAINTVISA.

2 Acadêmica do curso de Enfermagem da Faculdades Integradas da Vitória de Santo Antão FAINTVISA.

${ }^{3}$ Acadêmica do curso de Fisioterapia da Faculdade Santa Maria - FSM.

${ }^{4}$ Mestre em Ciências da Saúde pela Faculdade de Medicina do ABC, Santo André, SP. Docente da Faculdades Integradas da Vitória de Santo Antão - FAINTVISA. E-mail: anaceciliaamorim@hotmail.com.
} 
tuberculose, é necessário criar estratégias para diminuição desses números, precisa-se que a vigilância epidemiológica da tuberculose tenha o conhecimento dos casos da doença que ocorrem na população, permitindo a adoção de medidas que visam à interrupção da sua transmissão para indivíduos susceptíveis.

PALAVRAS CHAVE: Óbito. Saúde pública. Tuberculose.

ABSTRACT: OBJECTIVE: To analyze the incidence of tuberculosis deaths in the State of Pernambuco between 2015 and 2017. METHOD: The Tuberculosis Notification System (SINAN) database was evaluated in the State of Pernambuco from 2015 to 2017, provided by DATASUS / Ministry of Health. The data tabulation in the Department of Informatics of the Unified Health System (DATASUS) was carried out through SINAN, which is fed by the notification and investigation of cases of diseases and injuries of the national list of compulsorily notifiable diseases. A database was built in the SPSS Statistical Program version 22 with the variables included in the study. For the analysis of these data, percentage calculations were used to observe the dispersion among the variables collected, analyzed through a simple percentage. The information was analyzed through SPSS software version 22 for formatting the data. RESULTS: $A$ total of 16,933 cases of tuberculosis were reported in the state of Pernambuco in the period from 2015 to 2017. The municipality with the highest index of the disease was Recife, accounting for $45.75 \%$. The results showed that the sex most affected was the male sex (70.24\%), the age group most affected was between 20 and 39 years $(46.44 \%)$, the brown race predominated in case numbers (43.94\%), and evolution to death (5.82\%), $86.32 \%$ of the cases were classified as being pulmonary, and only $2.06 \%$ had both pulmonary and extrapulmonary forms. CONCLUSION: With the high number of confirmed cases of tuberculosis, it is necessary to create strategies to reduce these numbers, it is necessary that the epidemiological surveillance of tuberculosis be aware of the cases of the disease that occur in the population, allowing the adoption of measures aimed at the interruption of transmission to susceptible individuals.

KEYWORDS: Tuberculosis. Death. Public Health. 


\section{INTRODUÇÃO}

A tuberculose (TB) é uma doença bacteriana crônica, infecciosa, que afeta prioritariamente os pulmões, embora possa acometer outros órgãos e sistemas, transmissível de notificação compulsória que tem como agente etiológico $\mathrm{M}$. Tuberculosis, sendo descoberto em 1882 por Robert Kock, que isolou e descreveu o bacilo conseguindo cultiva-lo e reproduzir a doença em animais de laboratório (SOUZA JÚNIOR et al., 2018) 1 .

A principal forma de contágio da TB é através das vias aéreas. Podendo disseminar-se para diversos órgãos, caracterizando a forma extrapulmonar. Embora a tuberculose não seja uma doença nova, ainda se constitui como um dos principais problemas para saúde pública não só no Brasil, mas, a nível mundial (RODRIGUES et al., 2016) $)^{2}$.

Segundo estimativas da Organização Mundial da Saúde (OMS) em 2015, um total de 10,4 milhões de pessoas ficaram doentes com tuberculose ativa e 1,4 milhão morreu da doença, tornando-se a maior causa de morte mundial por um único agente infeccioso. Somada a isso, a resistência às drogas tem emergido recentemente como mais uma grande preocupação no enfrentamento da TB, ocasionando assim um aumento no número de casos, apresentando uma estimativa de 480 mil casos novos e 250 mil mortes no mundo, não sendo diferente no Brasil que teve em 20151.027 novos casos notificados no país (BRASIL, 2017; COÊLHO et al., 2017) $)^{3,4}$.

Pernambuco ocupa o $3^{\circ}$ lugar em taxa de incidência no âmbito nacional, a TB é a quarta causa de morte por doenças infecciosas e a primeira causa de morte em indivíduos com AIDS (PERNAMBUCO, 2012). . As pessoas portadoras do HIV apresentam ainda mais vulnerabilidade a adquirir a tuberculose, tendo em vista que estão interligadas, representando assim, cerca de $10 \%$ dos casos anuais de tuberculose. Isso se tornou um fator preocupante, pois a ligação do HIV com a tuberculose diminui as possibilidades de controle da tuberculose no mundo, tendo 
assim um aumento da incidência da tuberculose e, consequentemente, em casos de óbitos, aumentando o risco de infecção da tuberculose em até 19,0\% em relação a indivíduos que não tem a infecção pelo HIV (BRASIL, 2017)³.

Como medidas para o controle da tuberculose o Ministério da Saúde adota estratégias, entre elas a análise das tendências de morbidade e mortalidade da tuberculose, bem como os fatores associados (SANTOS et al., 2017) ${ }^{6}$. Desde a criação do Sistema de Informações de Agravos de Notificação (SINAN) a TB passou a ser uma doença de notificação compulsória. A alimentação regular da base de dados nacional pelos municípios, estados e Distrito Federal tornou-se obrigatória e o SINAN, atualmente, é a principal fonte de dados para a vigilância da TB (BARTHOLOMAY et al., 2014) ${ }^{7}$.

O diagnóstico da TB é fácil e o tratamento é gratuito e tem uma alta porcentagem de cura, contudo o número de óbitos por esta patologia ainda é considerado alto, sendo também o abandono ao tratamento outro fator preocupante, podendo atribuir esse abandono a diversos fatores como: longo período do tratamento, em média seis meses, toxicidade das drogas, dentre outros. Outro fator preocupante que dificulta o controle da tuberculose é a multirresistência ao tratamento, contribuindo assim que a doença evolua para um óbito, diminuindo mais ainda as chances de cura e controle da doença (SILVA et al., 2017) ${ }^{8}$.

Diante do exposto, fez-se necessário à realização desta pesquisa, objetivando conhecer o atual perfil de morbimortalidade da tuberculose em Pernambuco no ano de 2015 a 2017.

\section{MÉTODO}

Trata-se de um estudo epidemiológico comparativo do tipo descritivo de corte transversal, cujos dados foram coletados através do banco de dados secundários do Sistema de Informação de Agravos de Notificação (SINAN), fornecido pelo DATASUS/Ministério da Saúde. A população foi constituída pelos registros nas fichas de notificação para a doença "tuberculose" no Banco de Dados do SINAN em 
Pernambuco no ano de 2015 a 2017. O Departamento de Informática do Sistema Único de Saúde (DATASUS) disponibiliza informações que podem servir para subsidiar análises objetivas da situação sanitária, tomadas de decisão baseadas em evidências e elaboração de programas de ações de saúde.

A tabulação dos dados no Departamento de Informática do Sistema Único de Saúde (DATASUS) foi realizada através do SINAN, que é alimentado pela notificação e investigação de casos de doenças e agravos que constam da lista nacional de doenças de notificação compulsória, entre elas tuberculose, que é o alvo do estudo. As variáveis estudadas no banco de dados da tuberculose foram: sexo, faixa etária, raça, desfecho e forma de apresentação.

Foi construído um Banco de Dados no Programa Estatístico SPSS versão 22 com as variáveis incluídas no estudo. Para análise desses dados, foram utilizadas cálculos de porcentagem para observar a dispersão entre as variáveis coletadas, analisadas através de percentual simples. As informações foram analisadas por meio do programa SPSS versão 22 para a formatação dos dados.

\section{RESULTADOS}

Observou-se uma totalidade de 16.933 casos notificados de tuberculose no estado de Pernambuco no período de 2015 a 2017. O município com maior índice da doença foi Recife, caracterizando 45,75\%, em Jaboatão dos Guararapes com $6,49 \%$. Houve um expressivo quantitativo de casos no sexo masculino, atingindo $70,24 \%$ (Gráfico 1). 


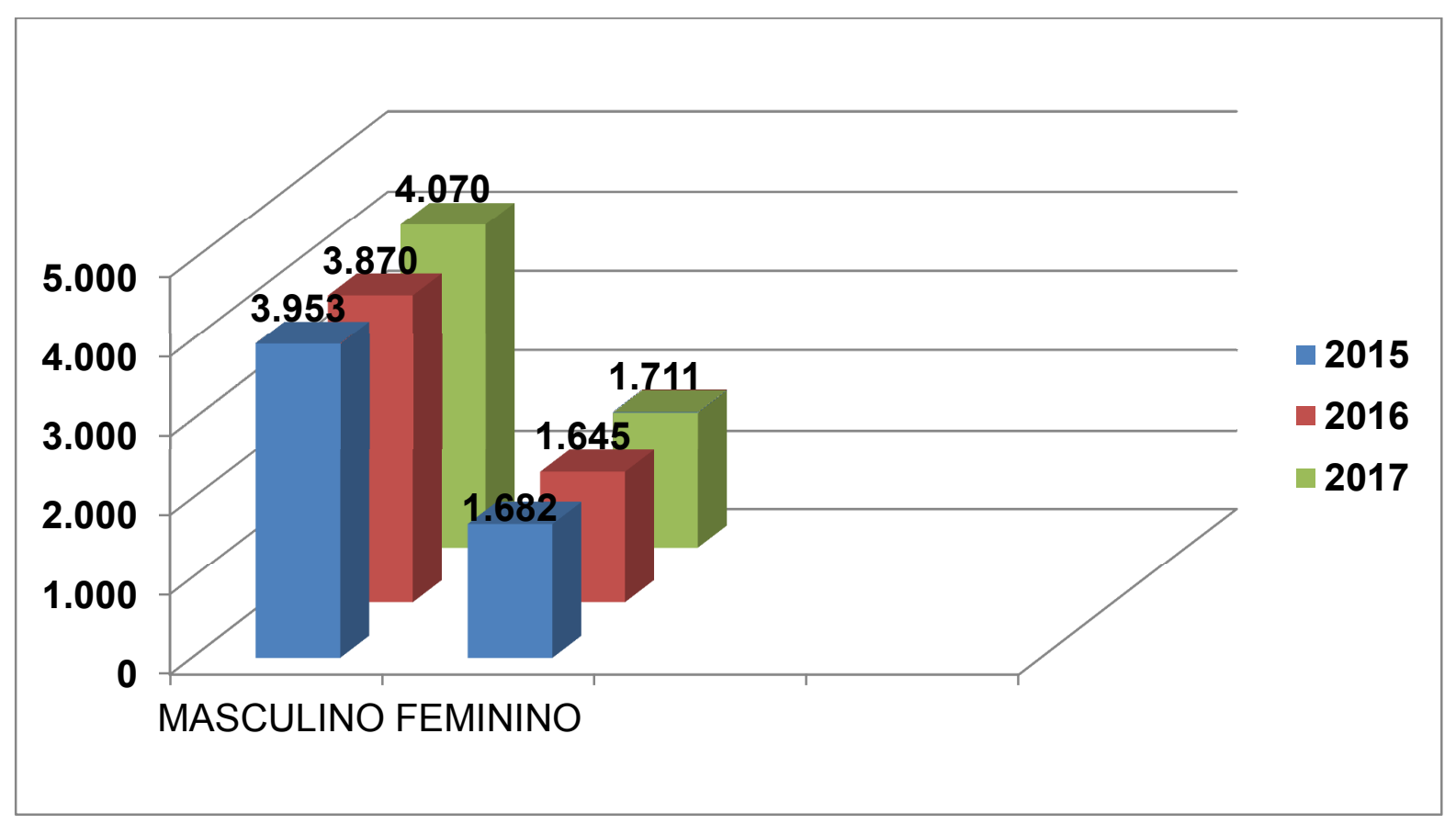

Gráfico 1 - Proporção de casos por sexo.

Fonte: Ministério da Saúde/SVS - Sistema de Informação de Agravos de Notificação - SINAN Net. Acesso em: 15/11/2018.

A raça parda com $43,94 \%$ foi a mais atingida no estado (Quadro 1 ).

Quadro 1 - Distribuição da tuberculose por raça em Pernambuco de 2015 a 2017.

\begin{tabular}{|c|c|c|c|c|c|}
\hline Raça & $\mathbf{2 0 1 5}$ & $\mathbf{2 0 1 6}$ & $\mathbf{2 0 1 7}$ & $\mathbf{N}^{\mathbf{2}}$ & $\mathbf{\%}$ \\
\hline Ign/Branco & 604 & 604 & 632 & 1.840 & 10,86 \\
\hline Branca & 1.051 & 1.028 & 985 & 3.064 & 18,09 \\
\hline Preta & 574 & 538 & 606 & 1.718 & 10,14 \\
\hline Amarela & 70 & 59 & 72 & 201 & 1,18 \\
\hline Parda & 3.319 & 3.263 & 3.467 & 10.049 & 59,34 \\
\hline Indígena & 19 & 23 & 19 & 61 & 0,36 \\
\hline TOTAL & $\mathbf{5 . 6 3 7}$ & $\mathbf{5 . 5 1 5}$ & $\mathbf{5 . 7 8 1}$ & $\mathbf{1 6 . 9 3 3}$ & $\mathbf{1 0 0}$ \\
\hline
\end{tabular}

Fonte: Ministério da Saúde/SVS - Sistema de Informação de Agravos de Notificação

- SINAN Net. Acesso em: 15/11/2018. 
Dentre a faixa etária a de 20-39 anos teve maior prevalência, representando $46,44 \%$ dos casos, em seguida aparece a de 40-59 anos com 31,92\% (Quadro 2).

Quadro 2 - Distribuição por faixa etária da tuberculose em Pernambuco de 2015 a 2017.

\begin{tabular}{|l|c|c|c|c|c|}
\hline \multicolumn{1}{|c|}{ Faixa etária } & $\mathbf{2 0 1 5}$ & $\mathbf{2 0 1 6}$ & $\mathbf{2 0 1 7}$ & $\mathbf{N}^{\circ}$ & $\mathbf{\%}$ \\
\hline Ign/Branco & 03 & 02 & 02 & 07 & 0.04 \\
\hline$<1$ Ano & 41 & 40 & 42 & 124 & 0,73 \\
\hline 01 a 4 & 20 & 31 & 35 & 86 & 0,50 \\
\hline 05 a 9 & 25 & 34 & 28 & 87 & 0,51 \\
\hline 10 a 14 & 57 & 34 & 70 & 161 & 0,95 \\
\hline 15 a 19 & 285 & 283 & 298 & 729 & 4,30 \\
\hline 20 a 39 & $\mathbf{2 . 5 8 7}$ & $\mathbf{2 . 5 2 8}$ & $\mathbf{2 . 6 7 1}$ & $\mathbf{7 . 7 8 6}$ & $\mathbf{4 5 , 9 8}$ \\
\hline 40 a 59 & $\mathbf{1 . 7 7 5}$ & $\mathbf{1 . 7 8 0}$ & $\mathbf{1 . 1 7 5}$ & $\mathbf{4 . 7 3 0}$ & $\mathbf{2 7 , 9 3}$ \\
\hline 60 a 64 & 284 & 272 & 297 & 853 & 5,03 \\
\hline 65 a 69 & 218 & 210 & 213 & 641 & 3,78 \\
70 a 79 & 251 & 219 & 235 & 705 & 4,16 \\
80 e mais & 91 & 82 & 115 & 288 & 1,70 \\
\hline \multicolumn{1}{|c|}{ TOTAL } & $\mathbf{5 . 6 3 7}$ & $\mathbf{5 . 5 1 5}$ & $\mathbf{5 . 7 8 1}$ & $\mathbf{1 6 . 9 3 3}$ & $\mathbf{1 0 0}$ \\
\hline
\end{tabular}

Fonte: Ministério da Saúde/SVS - Sistema de Informação de Agravos de Notificação - SINAN Net. Acesso em: 15/11/2018.

Em relação à evolução desses casos, 9,02\% abandonaram o tratamento, sendo $6,51 \%$ do abandono no sexo masculino, $86,32 \%$ foram classificadas como sendo a forma pulmonar, e apenas 2,06\% eram portadores das duas formas, pulmonar e extrapulmonar. Cerca de $11,84 \%$ tinham o HIV associado a tuberculose. Contudo se observou uma elevada prevalência no número de casos que evoluíram para cura, alcançando 53,68\%, seguido de 5,82\%, que evoluíram para óbito e 5,59\% de óbitos por outras causas (Quadro 3). 
Quadro 3 - Distribuição por situação de encerramento da tuberculose e distribuição de óbitos por tuberculose de acordo com o sexo em Pernambuco de 2015 a 2017.

\begin{tabular}{|c|c|c|c|c|c|}
\hline \multirow{2}{*}{ Vaiáveis } & 2015 & 2016 & 2017 & $\mathbf{N}^{\circ}$ & $\%$ \\
\hline & \multicolumn{5}{|c|}{ Encerramento } \\
\hline Ign/Branco & 102 & 193 & 1.916 & 2.211 & 13,05 \\
\hline Cura & 3.553 & 3.447 & 2.090 & 9.090 & 53,68 \\
\hline Abandono & 583 & 530 & 414 & 1.527 & 9,01 \\
\hline Óbito por TB & 326 & 296 & 354 & 976 & 5,76 \\
\hline Óbito por Outras C. & 340 & 327 & 265 & 932 & 5,50 \\
\hline Transferência & 653 & 637 & 698 & 1.988 & 11.74 \\
\hline Outros & 80 & 85 & 44 & 209 & 1,23 \\
\hline TOTAL & 5.637 & 5.515 & 5.781 & 16.933 & 100 \\
\hline \multicolumn{6}{|c|}{ Óbitos } \\
\hline Masculino & 247 & 214 & 277 & 738 & 4,35 \\
\hline Feminino & 77 & 79 & 92 & 248 & 1,47 \\
\hline TOTAL & 324 & 293 & 369 & 987 & 582 \\
\hline
\end{tabular}

Fonte: Ministério da Saúde/SVS - Sistema de Informação de Agravos de Notificação - SINAN Net. Acesso: 15/11/2018.

\section{DISCUSSÃO}

A tuberculose é uma doença infecciosa transmissível, por vias aéreas, que anualmente acomete milhares de pessoas. A multirresistência as drogas e a coinfecção pelo HIV dificultam mais ainda o tratamento. Embora o tratamento seja oferecido gratuitamente pelo Ministério da Saúde, ainda há elevadas taxas de incidências, e evoluções para óbitos decorrente das TB, podendo atribuir para o abandono ao tratamento ou até mesmo adesão inadequada (SOUSA et al., 2016) ${ }^{9}$. A TB prossegue como um perigo global à saúde, alcançando aproximadamente 10 milhões nos índices de incidências anuais, com evolução para óbito. Acabar com a TB é um desafio, estima-se que até 2030 haja uma redução de $90 \%$ das taxas de 
mortalidade e $80 \%$ das taxas de incidência de modo a eliminar a doença até 2050 (TRAJMAN; SARACENI; DUROVNI, 2018) ${ }^{10}$.

Pernambuco se apresenta em uma área bastante endêmica, podendo confirmar através dos elevados índices de notificação que aumenta a cada ano, isso também foi verificado por outros estudos realizados. Observou-se que o percentual de pacientes portadores da forma bacilífera pulmonar é alto, diferente de outros achados que encontraram prevalência na forma extrapulmonar. Este fato pode ser explicado pela diferença de estado pesquisado. A análise dos dados desta pesquisa permitiu constatar que mais da metade dos casos tiveram alta por cura, diferente de outros achados em que o índice de cura chega a menos de 40,0\% observados na pesquisa de Barbosa e Costa $(2014)^{11}$.

Barros et al. (2014) ${ }^{12}$ também observaram nos seus estudos que há uma maior prevalência da tuberculose no sexo masculino. Contudo, resultados diferentes foram percebidos por Costa et al. $(2014)^{13}$ que relatam não haver diferença significativa entre os sexos. No entanto, neste estudo observou-se uma diferença entre o sexo masculino e o feminino próximo a 40,0\%.

Em relação ao abandono do tratamento, notou-se que é um fato constante em todos os grupos, sugerindo que as ações de acompanhamento e tratamento não estão sendo positivas, podendo relacionar à não realização das baciloscopias de controle com esse abandono, além de fatores que estão profundamente ligados à situação socioeconômica da população acompanhada e à formação de um vínculo com a equipe que presta o atendimento ao paciente (MENDES et al., 2016) ${ }^{14}$.

Um fator que agrava a vulnerabilidade para a tuberculose é a presença do HIV, que devido ao fato de deprimir o sistema imune, o torna mais vulnerável a outras doença além de aumentar os riscos para o agravamento de patologia infecciosas como esta, uma vez que também se relaciona ao desenvolvimento de formas resistentes às drogas (SOUZA JÚNIOR et al.; 2018) ${ }^{1}$.

A tuberculose parece ser a causa principal de morte para os pacientes notificados com a doença. Aproximadamente um quarto morre em decorrência da TB no período de dois anos após o diagnóstico, embora exista tratamento eficaz amplamente disponibilizado na rede pública de saúde e de ser um óbito considerado evitável, ainda se tem um elevado número (ROCHA et al., 2015) ${ }^{15}$. 
Corroborando assim com nossos resultados que mostram que embora haja um expressivo número de óbito por outras causas, os óbitos por tuberculose ainda atingem um elevado índice, mesmo considerando que o preenchimento das informações é falho, ou os óbitos não são relacionado à doença, ou até mesmo ainda ficam em branco ou são ignorados chegando a 13,05\%.

Segundo Brasil $(2017)^{16}$ dentre as estratégias para o alcance das metas globais pactuadas, se faz necessário integrar sistematicamente um conjunto de ações de vigilância, que identifique os pontos de estrangulamento entre o processo de captação e notificação dos casos novos da doença. A informação em saúde deve ter como finalidade reduzir as incertezas e a identificação de situações prioritárias com vistas a subsidiar um planejamento adequado para a execução de ações que condicionem a realidade às transformações necessárias (SOUZA et al., 2015) ${ }^{17}$.

É de extrema relevância o monitoramento da evolução clínica da TB, através da realização de exames de acompanhamento. A baciloscopia de escarro deve ser realizada ao segundo, ao quarto e ao sexto mês de acompanhamento, sendo necessário pelo menos duas baciloscopias negativas para que o paciente receba alta, comprovando assim a cura, onde estas devem ser realizada no decorrer do acompanhamento e a outra ao final do tratamento. Comprovada a ausência de expectoração, recomenda-se periodicamente a radiografia de tórax, para acompanhar a evolução das lesões na forma pulmonar da doença. Algumas consequências podem ocorrer devido a não adesão das baciloscopias de controle como o retardo na identificação dos pacientes que não aderem ao tratamento, ou não respondem a terapia medicamentosa, podendo atrapalhar a detecção precoce de multirresistência bacteriana (BOSQUI et al., 2017; LIMA et al., 2016) ${ }^{18,19}$.

Diante do exposto se vê a relevância do planejamento de ações estratégicas para controle da tuberculose, com os altos números de casos confirmados de tuberculose, é necessário criar estratégias para diminuição desses números. Precisa-se que a vigilância epidemiológica da tuberculose tenha o conhecimento dos casos da doença que ocorrem na população, permitindo a adoção de medidas que visam à interrupção da sua transmissão para indivíduos susceptíveis.

Outro ponto relevante é o processo de produção das informações, pois se observa falhas no preenchimento da ficha de notificação em algumas variáveis, 
podendo assim comprometer um monitoramento seguro. Sugere-se por sua vez que haja cada vez mais campanhas educativas visando orientar a população sobre a doença, seu tratamento, bem como capacitação para equipe de saúde com o intuito de preencher as fichas de notificação adequadamente.

\section{CONCLUSÃO}

O homem por ser o estereótipo de virilidade e invulnerabilidade do ser que não adoece, não buscam os serviços de saúde, agravando assim o índice de morbimortalidade neste sexo. O presente estudo veio transcrever e comprovar que a população masculina é bastante acometida no tocante de doenças na região pernambucana. Entre os casos analisados, o sexo masculino predomina em casos notificados, e justamente na faixa etária compreendida entre 20 e 39 anos, atingindo a fase de maior vigor masculino.

\section{REFERÊNCIAS BIBLIOGRÁFICAS}

1. Souza Júnior EV, Nunes GA, Cruz DP, Boery EN, Boery RNSO. Internações hospitalares e impacto financeiro por tuberculose pulmonar na Bahia, Brasil. Rev. Enf. 2018; 35:1-14.

2. Rodrigues $A M X$, Silva KMR, Vieira MR, Macedo TF. Epidemiologia da tuberculose no Brasil nos últimos 10 anos. Rev. Enferm. UFPI. 2016; 5(2):75-79.

3. Ministério da Saúde. Secretaria de Vigilância em Saúde. Indicadores prioritários para o monitoramento do Plano Nacional pelo Fim da Tuberculose como Problema de Saúde Pública no Brasil. Boletim epidemiológico da Tuberculose [Online]. 2017;48,(8):1-11.

4. Coêlho AA, Souza MB, Costa NDL, Piuvezam G, Arcêncio RA, Uchôa SAC. Tuberculose no Brasil: relação entre porte populacional, estrutura e processo. Rev Enferm UERJ. 2017; $25: 1-8$.

5. Secretaria do Estado da Saúde. SES faz mobilização contra a tuberculose. 2012. Pernambuco: Secretaria do Estado da Saúde. http://portal.saude.pe.gov.br/noticias/ses-fazmobilizacao-contra-tuberculose (acesso em 20/Abr/ 2019).

6. Santos DT, Garcia MC, Costa AANF, Pieri FM, Meier DAP, Albanese SPR et al. Infecção latente por tuberculose entre pessoas com HIVIAIDS, fatores associados e progressão para doença ativa em município no Sul do Brasil. Cad. Saúde Pública 2017; 33(8):e00050916. 
7. Patricia Bartholomay, Gisele Pinto de Oliveira, Rejane Sobrino Pinheiro, Ana Maria Nogales Vasconcelos. Melhoria da qualidade das informações sobre tuberculose a partir do relacionamento entre bases de dados. Cad. Saúde Pública. 2014; 30(11): 2459-2469.

8. Silva FBG, Sodré MB, Santos FS, Costa ACPJ, Lobato JSM, Oliveira FJF et al. Perfil dos óbitos por tuberculose pulmonar em um município do nordeste brasileiro durante o período de 2005-2014. Arq. Cienc. Saúde UNIPAR. 2017; 21(3): 147-153.

9. Sousa LO, Mitano F, Lima MCRAd'A, Sicsú AN, Silva LMC, Palha PF. Terapia de curta duração da tuberculose: uma análise discursiva. Rev Bras Enferm [Internet]. 2016; 69 (6):115463.

10. Trajman A, Saraceni V, Durovni B. Os Objetivos do Desenvolvimento Sustentável e a tuberculose no Brasil: desafios e potencialidades. Cad. Saúde Pública 2018; 34(6): e00030318.

11. Barbosa IR, Costa ICC. Estudo epidemiológico da coinfecção tuberculose-hiv no nordeste do Brasil. Rev Patol Trop. 2014; 43 (1): 27-38.

12. Phelipe Gomes de Barros, Mayrla Lima Pinto, Talina Carla da Silva, Edwirde Luiz Silva, Tânia Maria Ribeiro Monteiro de Figueiredo. Perfil Epidemiológico dos casos de Tuberculose Extrapulmonar em um município do estado da Paraíba, 2001-2010. Cad. Saúde Colet., 2014, Rio de Janeiro, 22 (4): 343-50.

13. Ronaldo Rodrigues da Costa, Marcio Roberto Silva, Adalgiza da Silva Rocha, Kelly Cristina Ferreira Abi-Zaid, Antônio Augusto Fonseca Junior, Daniele Maria Knupp de Souza et al. Tuberculose: perfil epidemiológico em hospital referência no tratamento da doença. Rev Med Minas Gerais 2014; 24 (Supl 5): S57-S64.

14. Mendes AM, Bastos JL, Bresan D, Leite MS. Situação epidemiológica da tuberculose no Rio Grande do Sul: uma análise com base nos dados do SINAN entre 2003 e 2012 com foco nos povos indígenas. REV BRAS EPIDEMIOL JUL-SET 2016; 19(3): 658-669.

15. Rocha MS, Oliveira GP, Aguiar FP, Saraceni V, Pinheiro RS. Do que morrem os pacientes com tuberculose: causas múltiplas de morte de uma coorte de casos notificados e uma proposta de investigação de causas presumíveis. Cad. Saúde Pública; 2015; 31(4): 709721 ;

16. Ministério da Saúde. Brazil Free from Tuberculosis: National Plan to End TB as a Public Health Problem. Biblioteca Virtual em Saúde, Brasília [Online]. 2017; 1:1-54;

17. Souza MSPL, Aquino R, Pereira SM, Costa MCN, Barreto ML, Natividade M et al. Fatores associados ao acesso geográfico aos serviços de saúde por pessoas com tuberculose em três capitais do Nordeste brasileiro. Cad. Saúde Pública. 2015; 31(1): 111-120.

18. Larissa Rodrigues Bosqui, Suelen Santos Silva, Raquel Arruda Sanfelice, Milena Mengazzo Miranda-Sapla, Daniele Sapede Alvarenga, Bruno Bevenuto Lucas et al. Perfil clínico de pacientes com diagnóstico de tuberculose atendidos no Hospital Universitário de Londrina, Paraná. Semina: Ciências Biológicas e da Saúde; 2017; 38 (1): 89-98.

19. Lima LM, Harter J, Tomberg JO, Vieira DA, Antunes ML, Cardozo-Gonzales RI. Avaliação do acompanhamento e desfecho de casos de tuberculose em município do sul do Brasil. Rev Gaúcha Enferm. 2016 mar; 37(1): e51467. 